\title{
ON EXTREMAL MEASURES AND SUBSPACE DENSITY. II
}

\section{R. G. DOUGLAS}

In this note we continue the study begun in [1] of the relation between a measure being an extreme point in a certain convex set of probability measures and the density of a certain space of functions in the $L_{p}$-space of this measure. Our further thoughts on this subject are largely due to a recent communication of $\mathrm{J}$. Feldman.

Let $X$ be a locally compact Hausdorff space, and let $M(X)\left[M^{+}(X)\right]$ denote the space of finite [nonnegative], regular Borel measures defined on $X$. Let $F$ be a linear space of real-valued (not necessarily bounded) Borel functions defined on $X$ that contains the constant functions. For each $1 \leqq p<\infty[p=\infty]$ and each probability measure $\mu \in M^{+}(X)$ having the property that $\int_{X}|f|^{p} d \mu<\infty\left[\operatorname{ess}_{\mu}\right.$ sup $\left.|f|<\infty\right]$ for every $f \in F$, set

$$
\begin{array}{r}
E_{\mu}^{(p)}=\left\{\left.\gamma\left|\gamma \in M^{+}(X), \int_{X}\right| f\right|^{p} d \gamma<\infty[\operatorname{ess} \gamma \sup |f|<\infty]\right. \\
\text { and } \left.\int_{X} f d \mu=\int_{X} f d \gamma \forall f \in F\right\} .
\end{array}
$$

If $\mu \in E_{\mu}^{(p)}$, the space of functions $F$ can be identified (in a canonical way) as a subspace of $L_{p}(\mu)$ (this correspondence need not be one-to one).

We continue as in [1] to use the setting of Borel measures on locally compact spaces. The restatement of these results in the context of abstract measure spaces is obvious and we leave it to the reader.

We first extend Theorem 2 of [1] to include the case $p=\infty$.

TheOREM 3. If $F$ is also a vector lattice, then $F$ is weak*-dense in $L_{\infty}(\mu)$ if and only if $\mu$ is an extreme point of $E_{\mu}^{(\infty)}$.

Proof. If $\mu$ is not an extreme point of $E_{\mu}^{(\infty)}$, then there exist measures $\mu_{1}$ and $\mu_{2}$ in $E_{\mu}^{(\infty)}$ so that $\mu=\left(\mu_{1}+\mu_{2}\right) / 2$ and $\mu_{1} \neq \mu_{2}$. This implies that the Radon-Nikodym derivative of $\mu_{1}-\mu_{2}$ is in $(F)_{\infty \perp}$ so that $F$ is not weak*-dense in $L_{\infty}(\mu)$ (cf. [1, p. 243]).

If $\mu$ is an extreme point of $E_{\mu}^{(\infty)}$, then it is also an extreme point of $E_{\mu}^{(1)}$ so that $F$ is norm-dense in $L_{1}(\mu)$ by Theorem 1 of [1].

Let $g \in(F)_{\infty \perp} \subset L_{1}(\mu)$ and define

Received by the editors April 29, 1965. 


$$
k(x)= \begin{cases}1 & \text { if } g(x) \geqq 0, \\ -1 & \text { if } g(x)<0 .\end{cases}
$$

Then because $F$ is dense in $L_{1}(\mu)$ and is a vector lattice, there exists a sequence $\left\{f_{n}\right\}_{n=1}^{\infty}=1$ of functions in $F$ that converge pointwise a.e. to $k$ and so that $\left|f_{n}\right| \leqq 1$. The sequence $\left\{f_{n} g\right\}_{n=1}^{\infty}$ converges pointwise a.e. to $|g|$ and $\left|f_{n} g\right| \leqq|g|$; thus, from the dominated convergence theorem it follows that

$$
\int_{X}|g| d \mu=\lim _{n \rightarrow \infty} \int_{X} f_{n} g d \mu=0
$$

since $f_{n} \in F$ and $g \in(F)_{\infty \perp}$. Therefore $g=0$ a.e., $(F)_{\infty \perp}=\{0\}$, and $F$ is weak*-dense in $L_{\infty}(\mu)$.

The basic idea used in the preceding proof is due to Feldman.

We next consider the question of whether an extremal measure in $E_{\mu}^{(p)}$ is determined in some sense by its support. One has an intuitive feeling that the support of an extremal measure is "as small as possible." We show that this is indeed the case under certain additional assumptions.

THEOREM 4. If $F$ is weak*-dense in $L_{\infty}(\mu)$ and $\gamma \in M(X)$ so that $\int_{X} f d \mu=\int_{X} f d \gamma$ for $f \in F$ and $\gamma \prec \mu$, then $\gamma=\mu$.

Proof. Since $\gamma \prec \mu$, there exists a function $g \in L_{1}(\mu)$ by the RadonNikodym theorem so that $d \gamma=g d \mu$. Then $\int_{X}(1-g) f d \mu=\int_{X} f d \mu$ $-\int_{X} f d \gamma=0$ for $f \in F$ so that the function $1-g$ is orthogonal to $F$. Since $F$ is weak*-dense in $L_{\infty}(\mu)$ it follows that $1-g=0$ a.e. or that $\gamma=\mu$.

REMARK. Observe that the preceding result does not imply that measures $\mu_{1}$ and $\mu_{2}$ having the property that $F$ is weak*-dense in $L_{\infty}\left(\mu_{1}\right)$ and $L_{\infty}\left(\mu_{2}\right)$ are either equal or mutually singular. This is in fact not always true.

Corollary. If $F$ is also a vector lattice, $\mu$ is an extreme point of $E_{\mu}^{(1)}$ and $\gamma \in M(X)$ so that $\int_{X} f d \mu=\int_{X} f d \gamma$ for bounded $f \in F$, and $\gamma \prec \mu$, then $\gamma=\mu$.

Proof. If $F_{b}$ denotes the subspace of bounded functions in $F$, then $\mu$ is an extreme point of $E_{\mu}^{(1)}(F)$ if and only if it is an extreme point of $E_{\mu}^{(1)}\left(F_{b}\right)$. Now use Theorem 3 and 4 .

REMARK. Similar corollaries could be stated in which $F$ is replaced by a subalgebra of bounded real-valued Borel functions on $X$ that contains the constants since the closure of such in any of the mentioned topologies is a vector lattice. 
We conclude with a counterexample showing that Theorem 4 cannot be improved by replacing the weak*-density of $F$ in $L_{\infty}(\mu)$ by the norm-density of $F$ in $L_{p}(\mu)$ for all $1 \leqq p<\infty$. It is essentially the same example used in [1] and we take this opportunity to point out that in [1] the function $f$ must also satisfy the condition $\int_{X} f d \mu=0$ in order that the $F$ constructed contain the constant functions.

If $\mu$ is a measure that does not consist of a finite number of atoms, then there exists a nonnegative function $f$ in $L_{1}(\mu)$, that is not in $L_{p}(\mu)$ for $p>1$. Assume this for now; we justify it in the next paragraph. Assume further that $\int_{X} f d \mu=1$, and set $g=f-1$. If we let $F$ be the space of bounded Borel functions $k$ on $X$ having the property that $\int_{X} k g d \mu=0$, then $1 \in F, F$ is dense in $L_{p}(\mu)$ for $1 \leqq p<\infty$ but the measure $\gamma$ for which $d \gamma=f d \mu$ is in $E_{\mu}^{(\infty)}$ and $\gamma \prec \mu$.

To complete the argument we must show that $\bigcup_{p>1} L_{p}(\mu) \neq L_{1}(\mu)$ for a measure $\mu$ that does not consist of a finite number of atoms. One way to see this is to observe that it is sufficient to show that $L_{p}(\mu)$ for $p>1$ is a first category subset of $L_{1}(\mu)$. And this in turn follows from the fact that the unit ball of $L_{p}(\mu)$ is a closed subset of $L_{1}(\mu)$ which can contain no open set unless the $L_{p}$ and $L_{1}$ norms are equivalent. Since this happens only for the excluded case we are finished.

\section{REFERENCE}

1. R. G. Douglas, On extremal measures and subspace density, Michigan Math. J. 11 (1964), 243-246.

UNIVERSITY OF MichigAN 